\title{
PARP-1 inhibitors sensitize HNSCC cells to APR-246 by inactivation of thioredoxin reductase 1 (TrXR1) and promotion of ROS accumulation
}

\author{
Zhi-Xian Yin ${ }^{1, *}$, Wei Hang ${ }^{1,3, *}$, Gang Liu ${ }^{1}$, Yi-Shu Wang ${ }^{2}$, Xiang-Feng Shen ${ }^{2}$, Qian- \\ Hui Sun ${ }^{2}$, Dong-Dong Lí2, Yong-Ping Jian², Yang-He Zhang ${ }^{2}$, Cheng-Shi Quan², \\ Qinghua Zeng ${ }^{2,3}$, Yu-Lin Lí ${ }^{2}$, Rui-Xun Zhao ${ }^{3}$, Qiang Ding ${ }^{4}$ and Zhi-Xiang Xü ${ }^{2,3}$ \\ ${ }^{1}$ Department of Otorhinolaryngology Head and Neck Surgery, Tianjin Huanhu Hospital, Tianjin, China \\ ${ }^{2}$ Key Laboratory of Pathobiology, Ministry of Education, Norman Bethune College of Medicine, Jilin University, Changchun, \\ China \\ ${ }^{3}$ Division of Hematology and Oncology, Comprehensive Cancer Center, University of Alabama at Birmingham, Birmingham, \\ Alabama, USA \\ ${ }^{4}$ Department of Medicine, University of Alabama at Birmingham, Birmingham, Alabama, USA \\ *These authors have contributed equally to this work \\ Correspondence to: Zhi-Xian Yin, email: yinzhixian74@sohu.com \\ Zhi-Xiang XU, email: zhixiangxu08@gmail.com \\ Keywords: head and neck squamous cell carcinoma (HNSCC); PARP-1 inhibitors; p53 reactivators; reactive oxygen species (ROS); \\ thioredoxin reductase 1 (TrxR I) \\ Received: May 11,2017 Accepted: August 26, $2017 \quad$ Published: September 26, 2017 \\ Copyright: Yin et al. This is an open-access article distributed under the terms of the Creative Commons Attribution License 3.0 \\ (CC BY 3.0), which permits unrestricted use, distribution, and reproduction in any medium, provided the original author and source \\ are credited.
}

\section{ABSTRACT}

Head and neck squamous cell carcinoma (HNSCC) is the sixth most common cancer worldwide. Mutations of TP53 may reach $70 \%-85 \%$ in HNSCC patients without human papillomavirus (HPV) infection. Recurrence rate remains particularly high for HNSCC patients with mutations in the TP53 gene although patients are responsive to surgery, irradiation, and chemotherapy early in the treatment. p53-Reactivation and Induction of Massive Apoptosis-1 (PRIMA-1) and its methylated analogue PRIMA-1 ${ }^{\text {Met }}$ (also known as APR-246) are quinuclidine compounds that rescue the DNA-binding activity of mutant p53 (mut-p53) and restore the potential of wild-type p53. In the current report, we demonstrated that inhibition of poly (ADP-ribose) polymerase-1 (PARP-1) with 6(5H)-phenanthridinone (PHEN) and N-(6-Oxo-5,6dihydrophenanthridin-2-yl)-(N, N-dimethylamino) acetamide hydrochloride (PJ34) sensitizes UMSCC1, UMSCC14, and UMSCC17A, three HNSCC cell lines to the treatment of APR-246. PHEN enhances APR-246-induced apoptosis, but not programmed necrosis or autophagic cell death in HNSCC cells. The PARP-1 inhibition-induced sensitization of HNSCC cells to APR-246 is independent of TP53 mutation. Instead, PARP-1 inhibition promotes APR-246-facilitated inactivation of thioredoxin reductase 1 (TrxR1), leading to ROS accumulation and DNA damage. Overexpression of TrxR1 or application of antioxidant $\mathrm{N}$-acetyl-L-cysteine (NAC) depletes the ROS increase, reduces DNA damage, and decreases cell death triggered by APR-246/PHEN in HNSCC cells. Thus, we have characterized a new function of PARP-1 inhibitor in HNSCC cells by inactivation of TrXR1 and elevation of ROS and provide a novel therapeutic strategy for HNSCC by the combination of PARP-1 inhibitors and APR-246. 


\section{INTRODUCTION}

Head and neck squamous cell carcinoma (HNSCC) is the major type of head and neck cancer and ranks as one of the most common cancers worldwide. There are more than 700,000 new HNSCC cases diagnosed each year globally, with more than 350,000 deaths from the disease annually [1]. Despite the latest innovations in basic science and the improvement in clinical therapeutics, the overall 5-year survival rate for HNSCC remains low [2,3]. Development of new therapeutic drugs or exploration of combination for conventional therapy is urgently needed to effectively treat HNSCC.

Mutations of the TP53 tumor suppressor gene are the most frequent of all somatic genomic alterations in HNSCC. It is reported that mutations of TP53 may reach $70 \%-85 \%$ in HNSCC patients without human papillomavirus (HPV) infection [4-11]. Mutations of p53 lead to the loss of wild-type p53 function, the dominantnegative effect on the remaining wild-type p53, and even gaining oncogenic functions to promote tumorigenesis and progression [12-15]. Recurrence rate remains particularly high for HNSCC patients with mutations in the TP53 gene although patients are responsive to surgery, irradiation, and chemotherapy early in the treatment. These phenotypes render a potentially rationale for targeting TP53 mutations as a therapeutic intervention in HNSCC patients [16-23].

p53-Reactivation and Induction of Massive Apoptosis-1 (PRIMA-1) and its methylated analogue PRIMA-1 ${ }^{\text {Met }}$ (also known as APR-246) are quinuclidine compounds that rescue the DNA-binding activity of mutant p53 (mut-p53) and restore the potential of wild-type p53 $[16,21,24]$. PRIMA-1 and APR-246 are converted to methylene quinuclidinone (MQ), a Michael acceptor that can bind covalently to cysteines in mutant p53 and unfold wild type $\mathrm{p} 53$, hence restoring the activity of $\mathrm{p} 53[20,21$, 25]. Treatment with PRIMA-1 or APR246 up-regulates p53 target genes such as BAX, PUMA and NOXA and activates caspases $-2,-3$ and -9 [26]. Studies have revealed that MQ may also induce cell death independently of p53 in different tumor types [26]. It was found that MQ modifies and converts antioxidant thioredoxin reductase 1 (TrxR1) into pro-oxidant NADPH oxidase, leading to ROS production [27-29]. APR-246 may also reduces glutathione (GSH) content, hence further elevating the levels of intracellular ROS and eventually leading to DNA damage induced by the oxidative stress [30].

PARP-1 is the most abundant and active enzyme in the PARP family. PARP-1 binds to both single- and double-stranded DNA breaks, although its role in SSB repair via the BER pathway has been most clearly defined [31]. Inhibition of PARP leads to the accumulation of SSBs that become DSBs at replication forks. If the DSB repair mechanisms are impaired or otherwise insufficient, cells are unable to repair DNA damage. PARP inhibition in these cells will lead to a high degree of genomic instability and eventually cell death [32]. Thus, PARP inhibitors are actively investigated and show considerable promise as sensitizers for DNA damage agents and as therapeutic drugs for cancer patients bearing mutations in DNA repair genes or impaired DNA repair function [32-35]. In this study, we report a novel function of PARP inhibitor in the inactivation of TrxR1 and promoting APR-246-facilitated ROS accumulation and DNA damage, thereby enhancing APR-246-induced apoptosis in HNSCC cells.

\section{RESULTS}

\section{Inhibition of PARP sensitizes HNSCC cells to APR-246}

To determine the impact of PARP inhibition on TP53 reactivator-induced HNSCC cell viability, we treated UMSCC1, UMSCC14, and UMSCC-17A with APR-246, 6(5H)-phenanthridinone (PHEN, a PARP-1 inhibitor), or their combination for $72 \mathrm{~h}$. At dosages of 5 $\mu \mathrm{M}$ and $10 \mu \mathrm{M}$, PHEN alone only marginally induced cell death in HNSCC cells. Treatment with $10-40 \mu \mathrm{M}$ APR246 led to a modest reduction in the viability of HNSCC cells. Combination of PHEN with APR-246 resulted in a markedly enhanced overall cell death in a dose dependent manner and strikingly reduced IC50s (Figure 1A-1C, Supplementary Table 1). To assess the selectivity of the treatments to cancer (HNSCC) cells, we exposed primary MEFs to PHEN and/or APR-246. No noticeable cytotoxicity was observed in MEFs with the treatments at the dosages we detected (Figure 1D, Supplementary Table 1). Treatment with another PARP inhibitor, PJ-34, we obtained similar results in cell death after the treatment with or without APR-246 (Supplementary Figure 1).

\section{Inhibition of PARP-1 enhances APR-246-induced apoptosis in HNSCC cells}

To characterize the cell death induced by PARP1 and APR-246, we first determined whether the cell death is prompted by necrosis. Treatment with PHEN and/or APR-246 could not influence the expression of receptor interacting protein (RIP) kinases RIP1, RIP3, and phosphoglycerate mutase family member 5 (PGAM5), critical constituents triggering programmed necrosis (necroptosis) [36] (Figure 2A). PHEN and/or APR-246 associated cell death or cell viability was not affected by the pretreatment with necrostatin-1 (Nec-1), a selective RIP1 inhibitor [37] (Figure 2B), suggesting that PHEN and APR-246 at the current dosages $(10 \mathrm{uM}$ and $40 \mathrm{uM}$ respectively) are unable to induce necroptosis. Autophagy was reported to be associated with non-apoptotic programmed cell death and PRIMA-1 was reported to be able to induce autophagy at a relatively high concentration [38]. We asked whether PHEN could enhance APR-246induced cell death by promoting autophagy. Consistently, 
immunoblot assay showed that autophagy related gene 8 (ATG8), also called light chain 3 (LC3), underwent a conversion from the LC3-I isoform to the LC3-II isoform in APR-246-treated cells, indicating the induction of autophagy (Figure 2A). Autophagosome/autolysosome formation was observed in UMSCC14/GFP-LC3 cells treated with APR-246 (Figure 2C). However, PHEN could neither induce autophagy alone nor enhance APR246-induced autophagy (Figure 2A \& 2C), suggesting that PHEN-promoted cell death in APR-246-treated cells is not caused by autophagy.

PRIMA-1 and APR-246 were reported to induce apoptosis in vitro and in vivo [24-30]. To determine whether PHEN could enhance APR-246-induced cell death by promoting apoptosis, we detected apoptotic markers in the cell lysates. As shown in Figure 2A, the cleavage of PARP-1, caspase-9, and caspase-7 was markedly enhanced by the cotreatment with PHEN and APR-246. Detection of the cleaved DNA/histone complexes (nucleosomes) in the cells demonstrated the enrichment of nucleosomes in the cytoplasmic fraction of the cells co-treated with PHEN and APR-246, supporting the notion that the cell death is apoptosis (Figure 2D). To further confirm the induction of apoptosis by the cotreatment of PHEN and APR-246, cells were pretreated with benzyloxycarbonylvalyl-alanylaspartic acid (O-methyl)-fluoro-methylketone (zVAD-fmk), a pan-apoptotic inhibitor. As expected, the enrichment of nucleosomes in the cytoplasmic fraction of the cells cotreated with PHEN and APR-246 in the presence of zVADfmk was strikingly reduced although a small fraction of the cells still underwent cell death (Figure 2D), which may be due to additional non-apoptotic cell death. Taken together, we conclude that inhibition of PARP-1 enhances APR-246induced apoptosis in HNSCC cells.

\section{PARP-1 inhibition-induced sensitization of HNSCC cells to APR-246 is independent of TP53 mutation}

PRIMA-1 and APR-246 were initially screened and developed as re-activators of the mutant p53 gene [20, 25]. Recent studies showed that the compounds may possess a broad function in addition to the suppression of mutant $\mathrm{p} 53$ and reactivation of the $\mathrm{p} 53$ functions [28-30]. To determine whether the cell death from the cotreatment of PHEN and APR-246 is dependent of p53 mutation, we compared

A

UMSCC1



B

UMSCC14

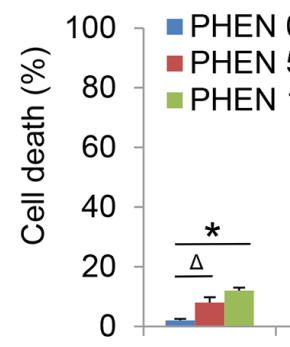

APR-246 $(\mu \mathrm{M}) \quad 0$

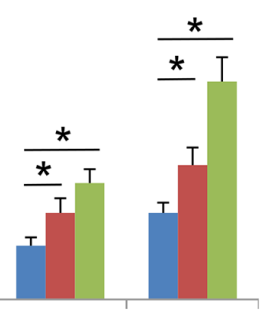

$20 \quad 40$
C UMSCC17A

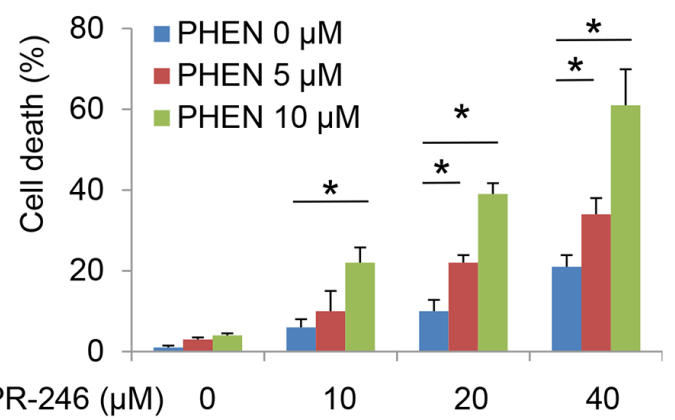

D

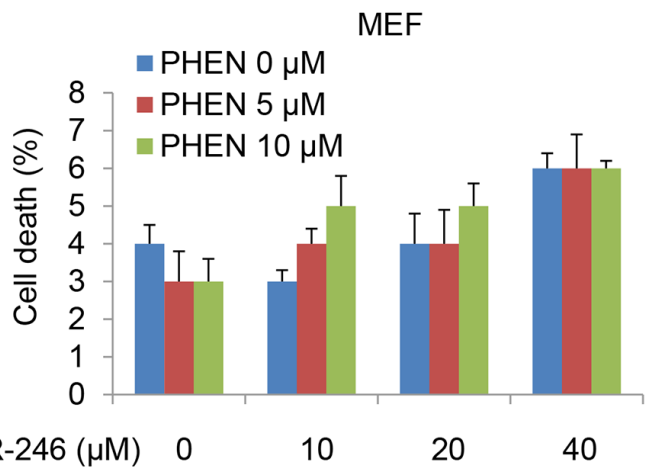

Figure 1: PARP Inhibitor PHEN sensitizes HNSCC cells to APR-246. Human HNSCC cell lines UMSCC1, UMSCC14, and UMSCC17A and primary MEFs were treated with different dosages of PHEN in the presence or absence of APR-246 for $72 \mathrm{~h}$. Cell death was determined by the trypan blue exclusion assay. The assays were performed in triplicate samples, and the results are representative of three independent experiments $\left({ }^{\Delta} \mathrm{P}<0.05 ;{ }^{*} \mathrm{P}<0.01\right.$ as compared with PHEN $0 \mu \mathrm{M}$ in each group). (A) UMSCC1; (B) UMSCC14 ; (C) UMSCC17A cells; and (D) MEFs. 
cell viability in UMSCC1 (p53 deficient), UMSCC14 (p53 mutation), and UMSCC17A (wild-type p53) under the treatment of both agents. As shown in Figure 1 and Supplemtary Figure 1, all the three cell lines responded to the cotreatment although p53 mutation UMSCC14 cells seemed to be more sensitive to the treatment. To further confirm the observation, we transduced wild-type and mutation p53 constructs to UMSCC1 cells (Figure 3A). Consistently, cells with wild-type and mutant p53 showed a similar response to the co-treatment (Figure 3B). Taken together, our results suggest that PARP inhibition-induced sensitization of HNSCC cells to APR-246 is independent of TP53 expression status.

\section{PARP-1 inhibitor promotes ROS accumulation in HNSCC cells}

PRIMA-1 is converted to methylene quinuclidinone (MQ), a Michael acceptor that can bind covalently to cysteines in mutant p53 and unfolded wild type p53, hence


Figure 2: Inhibition of PARP-1 enhances APR-246-induced apoptosis in HNSCC cells. (A) UMSCC14 cells were treated with $10 \mu \mathrm{M}$ PHEN and/or $40 \mu \mathrm{M}$ APR-246 for $24 \mathrm{~h}$. After the treatment, whole cell extracts were collected for the western blot analysis. (B) UMSCC14 cells were treated with $10 \mu \mathrm{M}$ PHEN and/or $40 \mu \mathrm{M}$ APR-246 in the presence or absence of $20 \mu \mathrm{M}$ necrostatin-1 (Nec-1) for $72 \mathrm{~h}$. After the treatment, cell death was determined as described in Figure 1. $\mathrm{n}=3$, NS = non-significant. (C) UMSCC14 cells were transfected with pcDNA3/GFP-LC3 for $24 \mathrm{~h}$. The cells were then treated with $10 \mu \mathrm{M}$ PHEN and/or $40 \mu \mathrm{M}$ APR-246 for additional $24 \mathrm{~h}$. Autophagosomes/autolysosomes (GFP-LC punctuates) were monitored under an Olympus IX51 fluorescence microscope. White arrows show the autophagosomes/autolysosomes. (D) UMSCC14 cells were pretreated with $20 \mu \mathrm{M} \mathrm{z}$-VAD-fmk for $6 \mathrm{~h}$ before addition of $10 \mu \mathrm{M}$ PHEN and/or $40 \mu \mathrm{M}$ APR-246 for $72 \mathrm{~h}$. After the treatment, cell apoptosis was quantified using a cell death ELISA kit (Roche Diagnostics) showing enrichment of nucleosomes in the cytoplasmic fraction of the cells. Values represent the mean \pm S.D. ${ }^{*} \mathrm{P}<0.01 ; \mathrm{n}=3$. 
restoring the activity of $\mathrm{p} 53$ [25]. Studies have revealed that MQ may also induce cell death independently of p53 in different tumor types [16]. One such mechanism is the induction of reactive oxygen species (ROS) by disturbing the cellular redox balance [27]. To determine whether PARP inhibition is able to promote ROS accumulation in APR-246 treated cells, we analyzed ROS levels in PHEN and/or APR-246 treated cells. Indeed, intracellular levels of ROS were increased in UMSCC14 cells exposed to APR-246. Treatment of PHEN also modestly increased the ROS level in the cells. Strikingly, co-treatment of PHEN and APR-246 led to a 3-fold increase in intracellular ROS (Figure 4). The antioxidant N-acetyl-L-cysteine (NAC) reduced ROS levels in PHEN and/or APR-246-treated cells (Figure 4). Together, our data support the notion that PHEN treatment enhances the ROS accumulation in cells exposed to APR-246.

\section{PARP-1 inhibitor augments APR-246-induced TrxR1 suppression}

Thioredoxin reductase 1 ( $\operatorname{Trx} R 1)$ is a cytoplasmic pyridine nucleotide oxidoreductase, which reduces thioredoxins (Trx) as well as other substrates. Trx executes antioxidant roles directly by reducing protein disulfides, as well as indirectly by donating reducing equivalents to peroxide scavenging enzymes such as peroxiredoxins or reductive repair enzymes such as methionine sulfoxide reductases or sulfiredoxin [39]. Peng et al. showed that APR-246/MQ is able to modify TrxR1 (antioxidant) and convert it to a dedicated NADPH oxidase (pro-oxidant), which can induce ROS production [27]. In addition, APR246 can deplete glutathione (GSH) content, thus further increasing intracellular ROS levels and ultimately leading to DNA damage induced by the oxidative stress [30]. To determine how PHEN exposure promotes the ROS accumulation in APR-246-treated cells, we determine TrxR1, NADPH oxidase, and GSH activity in the cells. Consistent with previous report, treatment with APR-246 reduced the activity of GSH and TrxR1 and modestly increased NADPH oxidase activity (Figure 5A-5C). Exposure to PHEN could not further manipulate GSH and NADPH oxidase activity (Figure 5A \& 5B), indicating that PHEN-promoted accumulation of ROS in APR-246treated cells may not be attributable to the dysregulation of GSH content and NADPH oxidase activity. PHEN alone
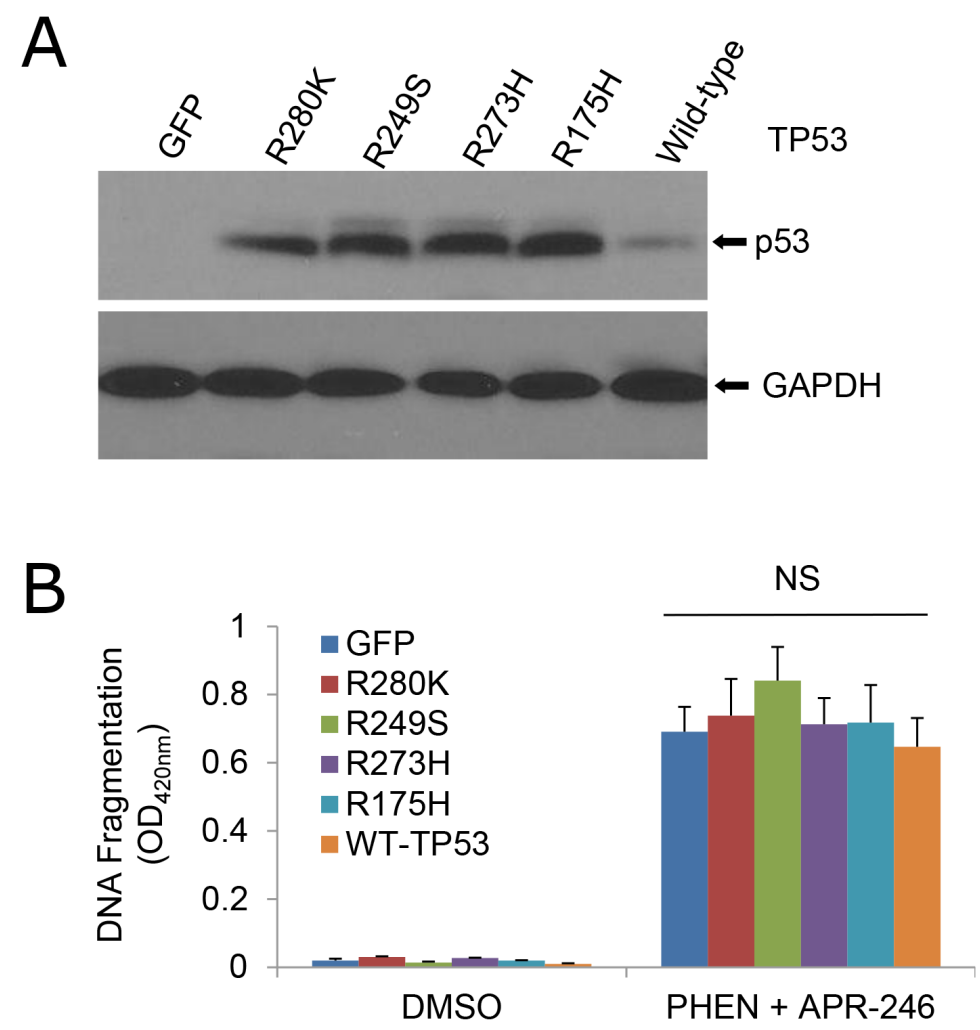

Figure 3: Sensitivity of cells to the cotreatment of PHEN and APR-246 is independent of TP53 mutation. UMSCC1 cells were infected with lentiviruses expressing TP53 mutants R280K, R249S, R273H, and R175H, wild-type TP53, or GFP (control). Cell transduction efficiency was at least $60 \%$ with the fluorescence microscopy analysis at $48 \mathrm{~h}$ after the infection. (A) Immunoblot analysis of p53 in the transduced cells. (B) Apoptosis in the cells treated with $10 \mu \mathrm{M}$ PHEN and $40 \mu \mathrm{M}$ APR-246 for additional $72 \mathrm{~h}$. Cell apoptosis was quantified using a cell death ELISA kit (Roche Diagnostics) showing enrichment of nucleosomes in the cytoplasmic fraction of the cells. The data represent the mean \pm S.D. NS: Non-significant. $\mathrm{n}=3$. 
or combined with APR-246 markedly reduced the activity of TrxR1 in UMSCC14 cells (Figure 5A). Interestingly, treatments of PHEN and/or APR-246 also moderately reduced the expression of TrxR1 (Figure 5D \& 5E), suggesting that the decreased TrxR1 activity upon the treatments in UMSCC14 cells may be due to both enzyme inhibition and decreased TrxR1 protein levels.

\section{TrxR1 is necessary for PARP-1 inhibitor - and APR-246 -induced ROS accumulation}

To validate the role of TrxR1 in PHEN and APR246-induced ROS accumulation, we knocked down TrxR1 in UMSCC14 cells followed by the cotreatment of PHEN and APR-246. It showed that PHEN could no longer further elevate ROS level in the presence or absence of APR-246 on the basis of an elevated ROS content as TrxR1 activity suppressed in the TrxR1-depleted cells (Figure 6A \& 6B, Supplementary Figure 2A). In contrast, NADPH oxidase activity was only marginally reduced in cells with or without the cotreatment (Supplementary Figure 2B). Overexpression of TrxR1 prevented the cells from PHEN-induced elevation of ROS in PHEN treatment alone or combination with APR-246 (Figure 6C \& 6D). Collectively, these results suggest that TrxR1 plays a critical role in PARP-1 inhibition-promoted elevation of ROS in HNSCC cells.

\section{APR-246 treatment induces DNA damage, which is enhanced by PARP inhibition}

PARP family proteins (mainly PARP-1 and PARP-2) participate in a physiological response against DNA damage and repair of single-strand DNA break (SSB)induced DNA damage [31]. Lack of PARP activity with genetic modification or inhibitors increases SSB count.
These unrepaired SSBs are converted into double-strand DNA breaks (DSBs) at replication fork [31]. If the DSB repair mechanisms are impaired or otherwise insufficient, cells are unable to repair DNA damage, which may lead to a high degree of genomic instability and cell death. To determine whether APR-246-initiated ROS accumulation leads to DNA damage, and more importantly, facilitated by cotreatment with PHEN, hence resulting in cell death, we exposed UMSCC14 cells to APR-246 and/or PHEN and measured the DNA damage by the comet assay. The tail moment that reflects the frequency of breaks was used to quantify DNA damage [40]. In APR-246- or PHENtreated cells, the level of damage was considerably higher than that in DMSO-treated (control) cells (Figure 7A \& 7B). Co-treatment with the two agents led to an obviously extended damaged DNA tail moment in cells (Figure 7A \& 7B). $\gamma$-H2AX, another DNA damage marker, was also increased in APR-246 or PHEN treated cells and enhanced by the co-treatment (Figure 7C). In keeping with the rescuing role of NAC treatment (Figure 4) or TrxR1 transduction (Figure 6D) against ROS accumulation, pretreatment with NAC or over-expression of TrxR1 reduced DNA damage (Figure 7C) and cell apoptosis triggered by APR-246/PHEN (Figure 7D \& 7E). Taken together, our results suggest that PHEN not only promotes the accumulation of ROS, but also facilitates DNA damage and cell death in APR-246-treated HNSCC cells.

\section{DISCUSSION}

Cisplatin-containing chemotherapy or its combination with radiation has become a standard care for patients with locally advanced HNSCC [41]. However, the recurrence rate of the disease is still high and the long-term survival in patients with advanced-stage head and neck cancer remains poor. Recent genomic data have revealed that up to $85 \%$

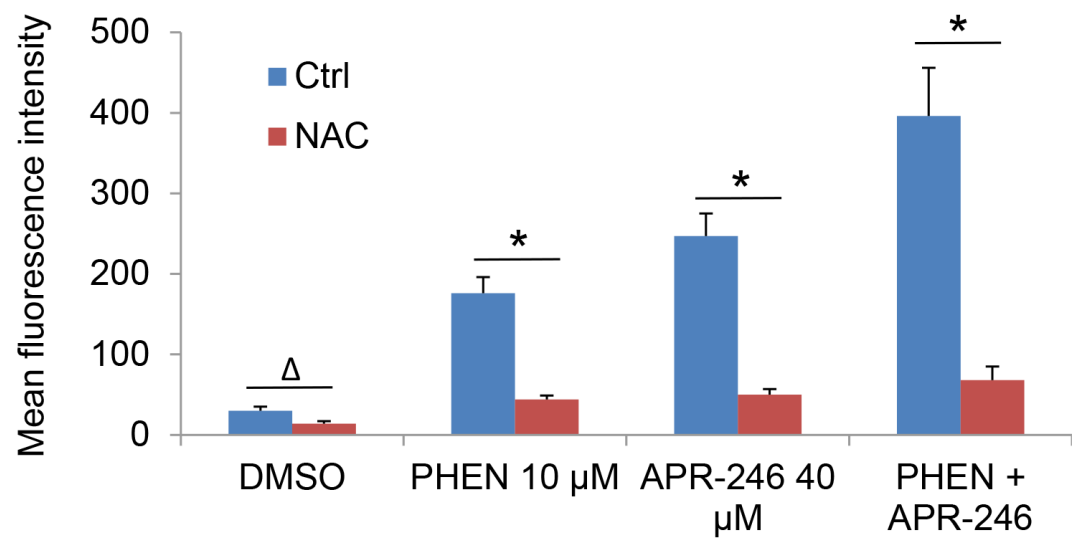

Figure 4: PARP-1 inhibition promotes the accumulation of ROS in APR-246-treated HNSCC cells, which is abrogated by pretreatment of antioxidant NAC. UMSCC14 cells were treated with $10 \mu \mathrm{M}$ PHEN and/or $40 \mu \mathrm{M}$ APR-246 in the presence or absence of $5 \mathrm{mM} \mathrm{NAC}$ for $24 \mathrm{~h}$. One hour prior to the termination of the treatment, $100 \mathrm{ng} / \mathrm{ml}$ dihydroethidium was added to the medium. The cells were collected, washed and analyzed by flow cytometry with the red laser channel (FL-3) using a FACscan analyzer. The data represent the mean \pm S.D. ${ }^{\Delta} \mathrm{P}<0.05,{ }^{*} \mathrm{P}<0.01, \mathrm{n}=3$. 
of non-HPV infection-induced HNSCC patients bear TP53 mutation, making it the most frequently mutated gene in HNSCC [4-10]. TP53 is a tumor suppressor, which plays a critical role in cell cycle arrest, senescence, activation of checkpoints after DNA damage and genotoxic stresses. Mutations of p53 may not only lead to the loss of wild-type p53 functions but also cause the dominant-negative effect on the remaining wild-type p53 and even gain oncogenic functions to promote tumorigenesis and progression [12]. TP53 mutation is associated with poor therapeutic response and decreased survival in HNSCC [13-15, 42]. Thus, an important objective in the clinic is to develop therapeutic strategies for overcoming chemotherapy resistance in patients with TP53 mutations.

With compound screening assays, a couple of agents have been developed in targeting TP53 mutations and/or restoring wild-type TP53 activities [22, 43, 44]. For example, PRIMA-1, APR-246, MIRA-1, CP-31398, and ellipticine restore p53 function on transcriptional transactivation and induce cell death preferentially in TP53 mutation carrying tumors [22]. RITA, nutlins, and HLI98 restore the tumor suppressive function of p53 by inhibiting MDM2-mediated p53 degradation in wildtype TP53-bearing tumors. Pharmacologic restoration of the p53 pathway induces cell-cycle arrest and massive apoptosis of tumors without causing adverse effects on normal cells. Thus, reconstitution of the p53 pathway is becoming one of the most exciting novel therapeutic strategies against cancer. In addition, the crucial role of p53 in DNA damage response makes it an ideal target for combination strategies between p53 activators and DNAdamaging agents or compounds interacting with DNA damage repair pathways [29].

PRIMA-1 and its analogue APR-246 have been reported to rescue the DNA-binding activity of mutant p53 (mut-p53) and restore the potential of wild-type p53. Several studies have shown the relevance of combining APR-246 with chemotherapeutic agents, resulting in
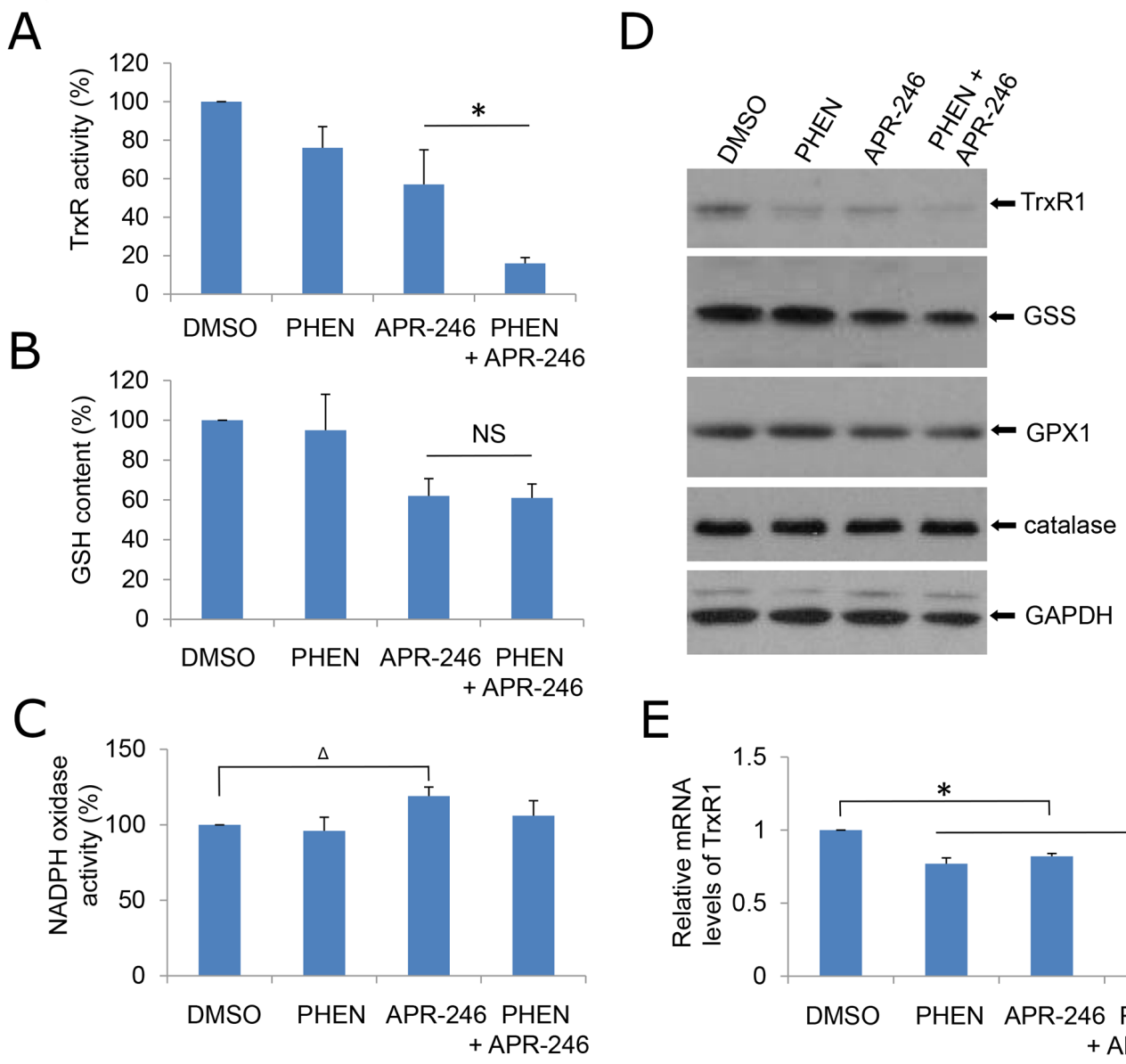

$\mathrm{E}$

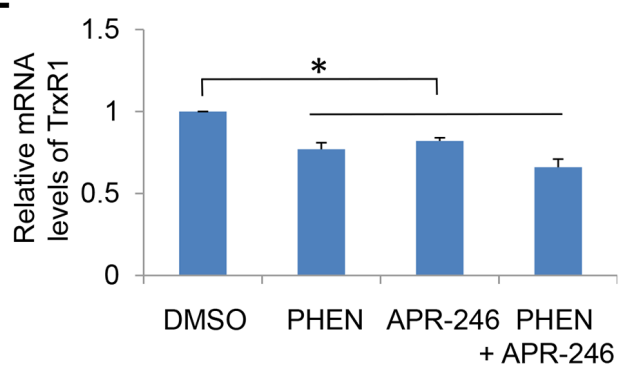

Figure 5: PHEN and ARP-246 suppress TrxR1. A total of 2 X $10^{5}$ cells were plated in six-well plates. The following day, cells were exposed to $10 \mu \mathrm{M}$ PHEN and/or $40 \mu \mathrm{M}$ APR-246 for $24 \mathrm{~h}$. The cells were then lysed. The clarified supernatants were used for the analysis of TrxR enzymatic activity (A), intracellular GSH concentration (B), NADPH oxidase activity (C), or immunoblot analysis (D) as described in Materials and Methods. (E) mRNA levels of TrxR1 in cells were determined by qPCR. The values in (A), (B), (C), and (E) were normalized to mg protein. The number in DMSO-treated cells was set as " $100 \%$ " or " 1 ". The data represent means \pm SD for 3 independent experiments. ${ }^{\Delta} \mathrm{P}<0.05,{ }^{*} \mathrm{P}<0.01$, NS: Non-significant. 
a synergistic effect and inhibition of chemotherapy resistance [22, 26, 29]. In addition, recent reports showed that PRIMA-1 and its analogue APR-246 may also bear functions on TP53 negative cells. PRIMA-1 and APR-246 are converted to methylene quinuclidinone (MQ), a Michael acceptor that covalently binds to cysteines in mutant p53 or unfolded wild type p53, thereby restoring its wild-type conformation $[14,21]$. It was reported that MQ modifies TrxR1 and converts this enzyme from a reductase to a NADPH oxidase that promotes ROS production, which may eventually contribute to PRIMA-1/APR-246-induced cell death and probably explains the observed effects of PRIMA-1/APR-246 on p53 null cancer cells [27]. Hypoxia is a causative factor for high ROS in tumor cells. It was found that hypoxia sensitized SKBR3 breast cancer cells carrying mutant p53 to PRIMA-1 [42]. The combination of PRIMA-1 with peroxidase increased apoptosis and induction of Puma and Mn-SOD in MCF-7 breast carcinoma cells overexpressing mutant p53 [45]. These results indicate that the antitumor activity of PRIMA-1 is influenced by hypoxia and suggest that PRIMA-1 may be useful for addressing chemoresistance in hypoxic tumors. In the current study, we found that APR-246 is cytotoxic not only to TP53 mutated UMSCC14 cells but to TP53 deficient UMSCC1 and TP53 wild-type UMSCC17A as well (Figure 1). Further validation revealed that APR-246 inactivated TrxR1 and promoted the accumulation of ROS (Figures 5 and 6). Interestingly, APR-246 only modestly increased NADPH oxidase activity, which could not explain a marked effect of APR-246 on ROS accumulation in the cells. Suppression of TrxR1-mediated reduction of Trx and the resultant antioxidant activity depletion may also contribute to the elevation of ROS in APR-246 treated UMSCC14 cells.

We demonstrated that PARP-1 inhibitors, PHEN and PJ34, markedly enhanced APR-246-induced cell death in HNSCC cells (Figure 1 and Supplementary Figure 1). Previous reports showed that APR-246 treatment leads to apoptosis associated with cleavage of PARP, which is involved in DNA repair and programmed cell death [46].
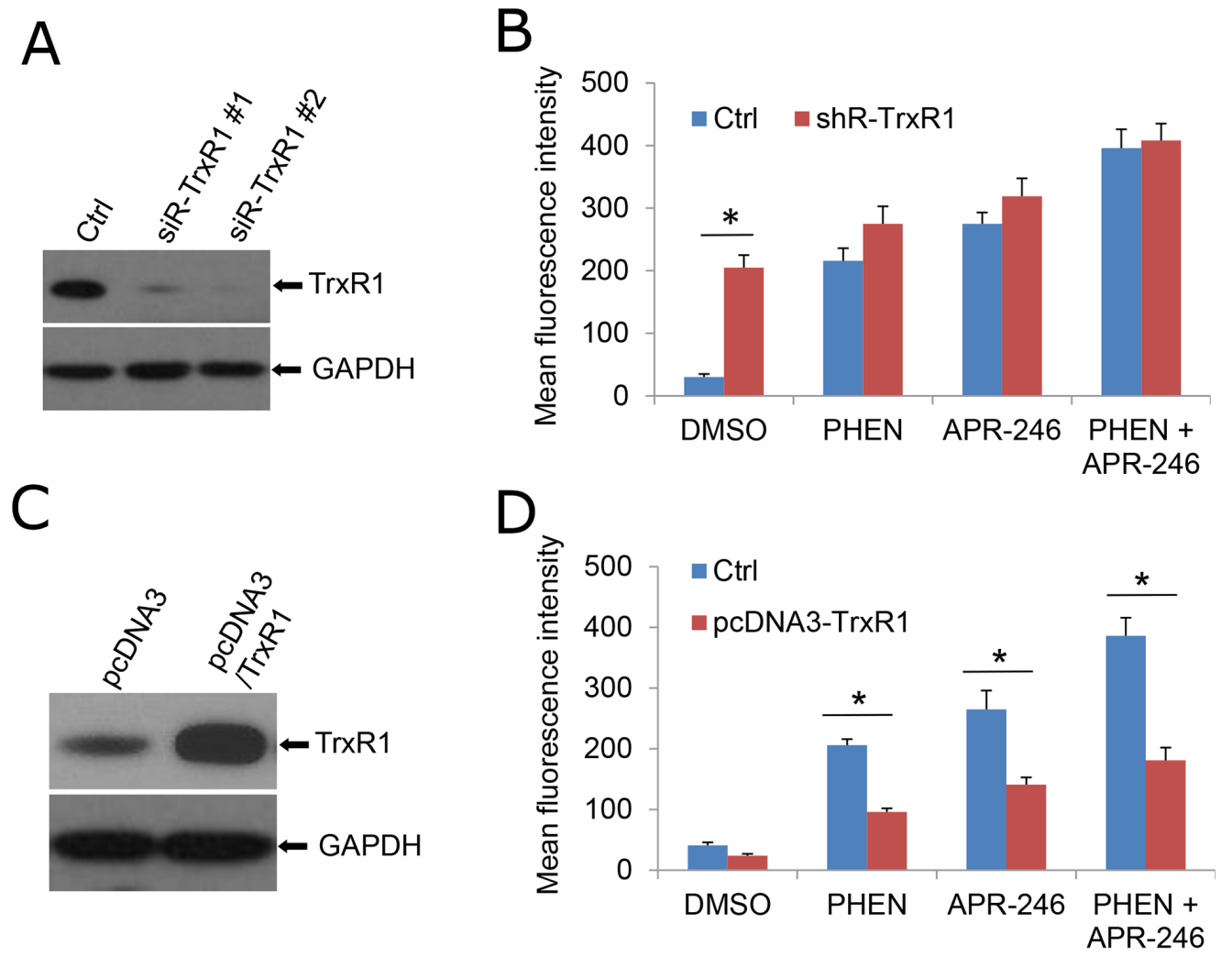

Figure 6: TrxR1 is necessary for PHEN- and APR-246-induced ROS accumulation. (A, B) Knockdown of TrxR1 increases intracellular ROS levels. Two duplexes of TrxR1 siRNA were transfected into UMSCC14 cells. Forty eight hours after the transfection, the cells were collected for the confirmation of knock-down by western blot (A) or exposed to $10 \mu \mathrm{M}$ PHEN and/or $40 \mu \mathrm{M}$ APR-246 for additional $24 \mathrm{~h}$ (B). Cells were then collected for the intracellular ROS determination. Measurements of ROS were performed as described in Figure 4. (C, D) Over-expression of TrxR1 reduces PHEN and APR-246 induced increase of ROS. TXNRD1 (TrxR1) gene was amplified with RT-PCR and cloned into pcDNA3 vector. pcDNA3/TrxR1 was transfected into UMSCC14 cells. Forty eight hours after the transfection, the cells were collected for the determination of TrxR1 expression by western blot (C) or exposed to $10 \mu \mathrm{M}$ PHEN and/ or $40 \mu \mathrm{M}$ APR-246 for additional $24 \mathrm{~h}$ (D). Cells were then collected for the intracellular ROS determination. Measurements of ROS were performed as described in Figure 4. ${ }^{*} \mathrm{P}<0.01, \mathrm{n}=3$. 
Thus, PARP-1 inhibitors may promote PRIMA-1/APR-246 facilitated cell death through the enhanced DNA damage [29]. In addition, in the current study, we characterize a new mechanism by which PARP-1 inhibitor enhances the killing effect of APR-246 on HNSCC cells. We found that PARP1 inhibitor, PHEN, suppressed the activity of TrxR1 and markedly increased the concentration of ROS in UMSCC14 cells (Figures 5 and 6). Overexpression of TrxR1 and application of antioxidant NAC significantly reduced ROS and cell death in HNSCC cells co-treated with APR-246 and PARP-1 inhibitor (Figures 4, 6C and 6D, 7D and 7E), further supporting our notion that TrxR1 is a target of PARP1 inhibitor PHEN. It is unknown how PARP-1 inhibitor suppresses TrxR1. It appears that both the activity and the expression of TrxR1 are involved (Figure 5A, 5D, and 5E). PARP participates in several cellular functions in addition
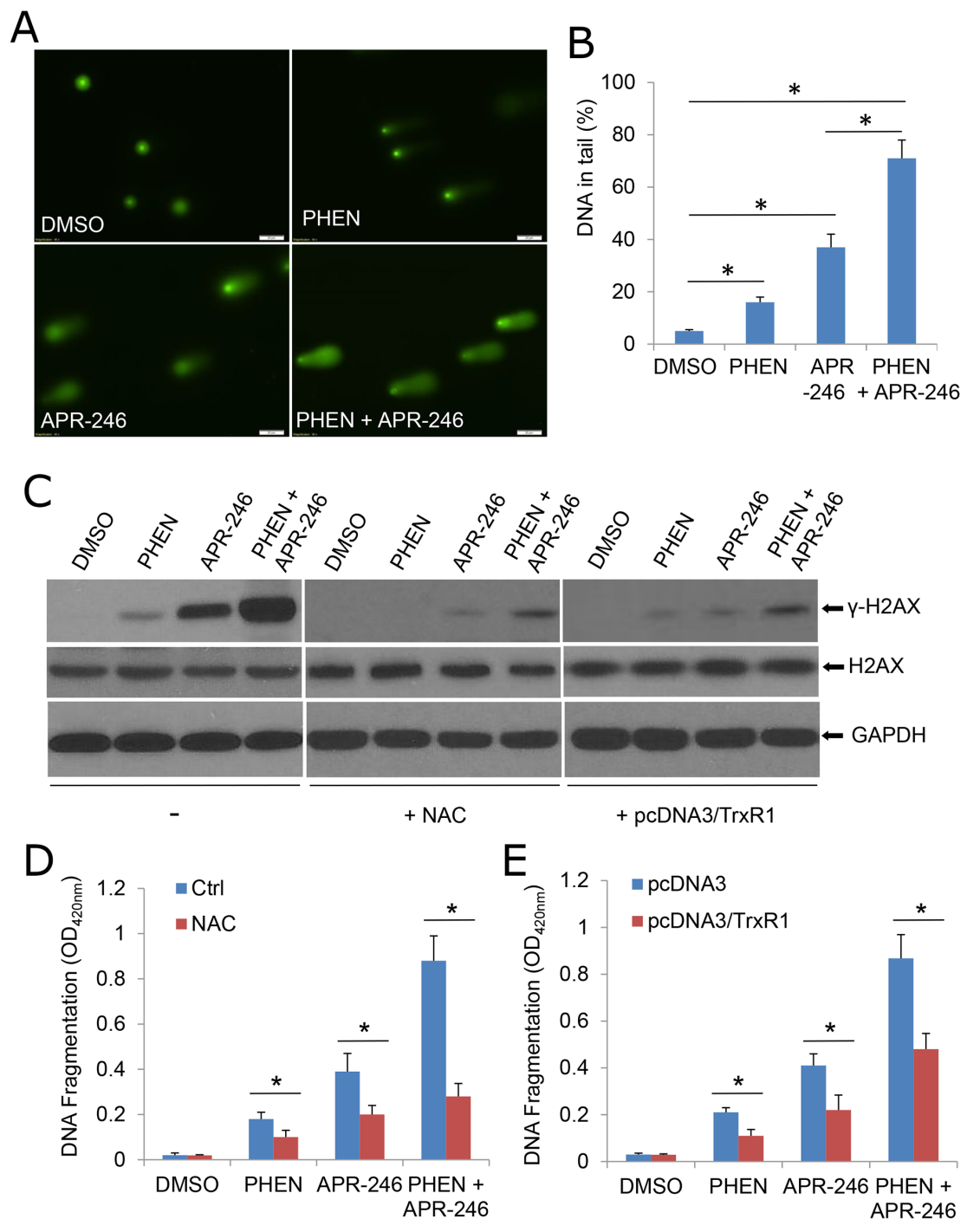

Figure 7: APR-246 treatment induces DNA damage, which is enhanced by PARP inhibition. (A, B) Comet assay demonstrating elevated DNA damage in cells treated with PHEN and/or APR-246. UMSCC14 cells were treated with $10 \mu \mathrm{M}$ PHEN and/or $40 \mu \mathrm{M}$ APR-246 for $24 \mathrm{~h}$. The cells were then trypsinized and washed with PBS. Two thousand cells were mixed with $100 \mu \mathrm{l}$ low melting agarose for alkaline comet assay. Cells in the gel were stained and visualized with epifluorescence microscopy (A). (B) Percentage of DNAs in the tail (damaged DNA) was calculated. ${ }^{*} \mathrm{P}<0.01, \mathrm{n}=3$. (C) DNA damages were markedly escalated in PHEN- and APR-246-treated cells. Antioxidant NAC and over-expression of TrxR1 reduce above agents-induced DNA damage. The indicated UMSCC14 cells were exposed to $10 \mu \mathrm{M}$ PHEN and/or $40 \mu \mathrm{M}$ APR-246 for $24 \mathrm{~h}$. Cell lysates were collected for western blot analysis. GAPDH serves as loading control. (D, E) NAC pretreatment and over-expression of TrxR1 reduce PHEN- and APR-246- induced apoptosis. UMSCC14 cells were pretreated with $5 \mathrm{mM} \mathrm{NAC}$ for $6 \mathrm{~h}$ (D) or transfected with pcDNA3/TrxR1 as described in Figure 6C and 6D (E). The cells were then exposed to $10 \mu \mathrm{M}$ PHEN and/or $40 \mu \mathrm{M}$ APR-246 for $48 \mathrm{~h}$. After the treatment, cell apoptosis was quantified using a cell death ELISA kit (Roche Diagnostics) showing enrichment of nucleosomes in the cytoplasmic fraction of the cells. Data represent the mean $\pm \mathrm{S} . \mathrm{D}$. ${ }^{*} \mathrm{P}<$ $0.01, \mathrm{n}=3$. 
to DNA repair, such as inflammation, DNA methylation, chromatin modification and cell death [31]. PARP-1 regulates the chromatin structure, which inherently affects the accessibility of DNA. It has been demonstrated that PARP-1 can simultaneously bind multiple nucleosomes, which results in de-condensation of the compact chromatin structure that represses gene transcription [31]. Thus, PARP-1 inhibition may lead to the suppression of multiple transcriptional factors, such as NF- $\kappa \mathrm{B}$ and Nrf2 (Nuclear factor erythroid2-related factor 2). It was reported that TrxR1 and Nrf2 can reciprocally regulate each other [47-49]. Cadmium-induced TrxR1 gene expression is mediated by the activation of Nrf2 transcription factor and its binding to ARE in the TrxR1 gene promoter [49]. Thus, Nrf2 may be a potential node transducing the action of PARP-1 inhibitor to TrxR1.

Under physiologic conditions, normal cells maintain redox homeostasis by controlling the proper balance between ROS generation and elimination. The redox dynamics may fluctuate within a tolerable range. An increase of ROS may promote cell proliferation and survival, as in the case of many cancer cells. However, when the increase of ROS reaches a critical level (the threshold), it may overwhelm the cellular antioxidant capacity and trigger the cell-death process [50]. Cell death induced by many chemotherapeutic agents, histone deacetylase inhibitors, proteasome inhibitors, redox cycling agents, and PARP-1 inhibitors and APR-246 (this paper), all appear to increase oxidant stress in cells [50]. This common effect suggests that neoplastic cells may be more vulnerable to oxidant stress because they function with a heightened basal level of ROS-mediated signaling, which is required for the increased rate of growth [51]. Therefore, addition of an agent that increases ROS generation, or that decreases ROS scavenging capacity, may push the ROS level in the tumor to reach a threshold that may overwhelm the cellular antioxidant capacity and trigger the cell-death process [50]. Indeed, this concept was verified by the combined application of APR-246 and PARP-1 inhibitor (Figures 1 and 4). By contrast, normal cells exhibit a smaller increase in oxidant stress because their baseline levels of oxidant signaling are smaller, so the elevation of ROS or depletion of antioxidants presumably has less severe consequences for the cellular oxidative-redox environment [52]. Therefore, to the extent that ROS toxicity induced by certain chemotherapeutic agents or natural compounds can be an effective means of selectively eradicating malignant cells, it is useful to consider the most effective way to exploit this strategy in the future.

\section{MATERIALS AND METHODS}

\section{Cell culture and drug treatment}

Human HNSCC cell lines UMSCC1, UMSCC14, and UMSCC17 were originally from Thomas Carey at the University of Michigan and maintained in the lab [53].
Mouse embryonic fibroblasts were isolated from C57BL6 mice. Cells were cultured in DMEM supplemented with $10 \%$ fetal bovine serum (Atlanta Biologicals, Atlanta, GA) and maintained at $37^{\circ} \mathrm{C}$ under a humidified $5 \%$ $\mathrm{CO}_{2}$ atmosphere. Poly (ADP-ribose) polymerase (PARP) inhibitors 6(5H)-phenanthridinone (PHEN) and N-(6-Oxo5,6-dihydrophenanthridin-2-yl)-(N, N-dimethylamino) acetamide hydrochloride (PJ34), and APR-246 were purchased from Sigma-Aldrich (St. Louis, MO). zVADfmk was purchased from Biomol International (Plymouth Meeting, PA).

\section{Plasmids, gene transfection, and establishment of over-expression stable cell lines}

Wild-type and mutation p53 constructs (R280K, $\mathrm{R} 249 \mathrm{~S}, \mathrm{R} 273 \mathrm{H}$, and $\mathrm{R} 175 \mathrm{H}$ ) were purchased from Addgene and sub-cloned into lentivirus vector [54]. UMSCC1 cells were transduced with lentiviruses expressing these mutations and selected with puromycin. Scramble siRNA and siRNA duplexes to human TrxR1 were ordered from Thermo Scientific (Lafayette, CO) and transfected to the cells with siRNA transfectant \#2 (Thermo Scientific).

\section{Cell viability and cell death assay}

Cell viability was measured by the MTT assay as described previously. ${ }^{38}$ Cell death was determined by trypan blue (Sigma-Aldrich, MO) exclusion assay. Cell apoptosis was quantified using a cell death ELISA kit (Roche Diagnostics) showing enrichment of nucleosomes in the cytoplasmic fraction of the cells.

\section{Fluorescence microscopy}

UMSCC14 cells were grown in a six-well plate and transfected with pcDNA3/GFP-LC3 for $24 \mathrm{~h}$. The cells were then treated with PHEN and/or APR-246. The autophagosomes/autolysosomes (GFP-LC3 punctuates) were monitored under an Olympus IX51 fluorescence microscope (Center Valley, PA, USA).

\section{Antibodies and western blot}

After the indicated treatments, cells were lysed by icecold RIPA buffer containing proteasome-inhibitor cocktail (Cell Signaling, Danvers, MA). Protein samples were separated by SDS-PAGE, transferred onto a polyvinylidene difluride membrane (Thermo Scientific, Rockford, IL), and probed with an appropriate antibody. Antibodies against caspase-7, caspase-9, p53 were purchased from BD Pharmingen (San Diego, CA). Anti- human RIP1, RIP3, GPX1, Catalase, $\gamma-\mathrm{H} 2 \mathrm{AX}, \mathrm{H} 2 \mathrm{AX}$, and PGAM5 antibodies were from Abcam Inc. (Cambridge, MA). AntiLC3 antibody was purchased from Novus Biological Inc. (Littleton, CO). TrxR1 antibody (B-2) and glutathione 
synthetase (GSS) antibody (C-5) were from Santa Cruz Biotechnology (Santa Cruz, CA). A total of $30 \mu \mathrm{g}$ protein was used for the immunoblotting, unless otherwise indicated. GAPDH was used for the loading control.

\section{Detection of intracellular ROS}

One hour prior to the termination of the treatment, $100 \mathrm{ng} / \mathrm{ml}$ dihydroethidium was added to the medium. The cells were harvested, washed, and analyzed by flow cytometry with the red laser channel (FL-3) using a FACscan analyzer [40]. ROS were also determined using a 96-well plate-based intracellular ROS assay as we previously reported [40].

\section{Measurement of enzyme activity of TrxR, NADPH oxidase, and GSH}

A total of $2 \times 10^{5}$ cells were plated in six-well plates. After 24 h-incubation, the cells were treated and lysed, and the clarified supernatants were used for the analysis of TrxR enzymatic activity, NADPH oxidase activity, intracellular GSH, and determination of total protein concentrations. Intracellular TrxR activity was measured using a Thioredoxin Reductase Assay Kit (Colorimetric) (ab83463) following the instructions of the manufacturer. The NADPH oxidase activity was meaured by the juglone-coupled assay as previously reported $[27,55]$. A final concentration of $12.5 \mathrm{nM}$ modified TrxR1, $200 \mathrm{mM}$ NADPH and $50 \mathrm{mM}$ juglone in TE buffer was used. The reaction was assessed by measuring NADPH consumption through the decrease of absorbance at $340 \mathrm{~nm}$. Intracellular contents of GSH were determined using a GSH/GSSG Ratio Detection Assay Kit (Fluorometric-Green) (ab138881) according to the instructions of the manufacturer. Total protein concentrations were determined with a Bradford reagent kit (Bio-Rad Laboratories, Hercules, California).

\section{Real-time quantitative PCR}

Total RNA was isolated with TRIZOL reagent (Life Technology, Grand Island, NY) and Direct-zol RNA miniPrep kit (Zymo Research, Irvine, CA). Comparative real-time polymerase chain reaction (qPCR) using SYBR Green SuperMix (Invitrogen, USA) was performed in a 96well plate and was run in a 7500 Real-Time PCR System (Applied Biosystems) at $95^{\circ} \mathrm{C}$ for 10 minutes, followed by 40 cycles of $95^{\circ} \mathrm{C}$ for 15 seconds and $57^{\circ} \mathrm{C}$ for 1 minute. Each sample was analyzed in duplicate or triplicate.

\section{Alkaline comet assay}

We followed the method for alkaline comet assay that we reported previously [40]. The cells were treated and run in alkaline buffer for $20 \mathrm{~min}$, fixed, stained, and examined under the fluorescence microscope.

\section{Statistical analysis}

Data were obtained from at least three independent experiments performed in triplicate and expressed as the means \pm SD. Statistical significance was determined using the student's $t$-test analysis, and $\mathrm{p}$ value $<0.05$ was considered significant.

\section{CONFLICTS OF INTEREST}

The authors declare no conflicts of interest.

\section{FUNDING}

The work was supported by grants from the National Natural Science Foundation of China No. 81573087, No. 81772924, No. 81271853, and No. 81272243 and the U.S. National Institutes of Health (R01CA133053).

\section{REFERENCES}

1. Torre LA, Bray F, Siegel RL, Ferlay J, Lortet-Tieulent J, Jemal A. Global cancer statistics, 2012. CA Cancer J Clin. 2015; 65: 87-108.

2. Sacco AG, Cohen EE. Current treatment options for recurrent or metastatic head and neck squamous cell carcinoma. J Clin Oncol. 2015; 33: 3305-13.

3. Skinner HD, Sandulache VC, Ow TJ, Meyn RE, Yordy JS, Beadle BM, Fitzgerald AL, Giri U, Ang KK, Myers JN. TP53 disruptive mutations lead to head and neck cancer treatment failure through inhibition of radiation-induced senescence. Clin Cancer Res. 2012; 18: 290-300.

4. Cancer Genome Atlas Network. Comprehensive genomic characterization of head and neck squamous cell carcinomas. Nature. 2015; 517: 576-82.

5. Parfenov M, Pedamallu CS, Gehlenborg N, Freeman SS, Danilova L, Bristow CA, Lee S, Hadjipanayis AG, Ivanova EV, Wilkerson MD, Protopopov A, Yang L, Seth S, et al. Characterization of HPV and host genome interactions in primary head and neck cancers. Proc Natl Acad Sci U S A. 2014; 111: 15544-9.

6. Hedberg ML, Goh G, Chiosea SI, Bauman JE, Freilino ML, Zeng Y, Wang L, Diergaarde BB, Gooding WE, Lui VW, Herbst RS, Lifton RP, Grandis JR. Genetic landscape of metastatic and recurrent head and necksquamous cell carcinoma. J Clin Invest. 2016; 126: 169-80.

7. Sun W, Califano JA. Sequencing the head and neck cancer genome: implications for therapy. Ann N Y Acad Sci. 2014; 1333: 33-42.

8. Iglesias-Bartolome R, Martin D, Gutkind JS. Exploiting the head and neck cancer oncogenome: widespread PI3KmTOR pathway alterations and novel molecular targets. Cancer Discov. 2013; 3: 722-5. 
9. Stransky N, Egloff AM, Tward AD, Kostic AD, Cibulskis K, Sivachenko A, Kryukov GV, Lawrence MS, Sougnez C, McKenna A, Shefler E, Ramos AH, Stojanov P, et al. The mutational landscape of head and neck squamous cell carcinoma. Science. 2011; 333: 1157-60.

10. Sepiashvili L, Bruce JP, Huang SH, O’Sullivan B, Liu FF, Kislinger T. Novel insights into head and neck cancer using next-generation "omic" technologies. Cancer Res. 2015; 75: 480-6.

11. Agrawal N, Frederick MJ, Pickering CR, Bettegowda C, Chang K, Li RJ, Fakhry C, Xie TX, Zhang J, Wang J, Zhang N, El-Naggar AK, Jasser SA, et al. Exome sequencing of head and neck squamous cell carcinoma reveals inactivating mutations in NOTCH1. Science. 2011; 333: 1154-7.

12. Zhou G, Wang J, Zhao M, Xie TX, Tanaka N, Sano D, Patel AA, Ward AM, Sandulache VC, Jasser SA, Skinner HD, Fitzgerald AL, Osman AA, et al. Gain-of-function mutant p53 promotes cell growth and cancer cell metabolism via inhibition of AMPK activation. Mol Cell. 2014; 54: 960-74.

13. Zhou G, Liu Z, Myers JN. TP53 Mutations in head and neck squamous cell carcinoma and their impact on disease progression and treatment response. J Cell Biochem. 2016; 117: 2682-92.

14. Soussi T, Wiman KG. TP53: an oncogene in disguise. Cell Death Differ. 2015; 22: 1239-49.

15. Poeta ML, Manola J, Goldwasser MA, Forastiere A, Benoit N, Califano JA, Ridge JA, Goodwin J, Kenady D, Saunders J, Westra W, Sidransky D, Koch WM. TP53 mutations and survival in squamous-cell carcinoma of the head and neck. N Engl J Med. 2007; 357: 2552-61.

16. Yu X, Vazquez A, Levine AJ, Carpizo DR. Allele-specific p53 mutant reactivation. Cancer Cell. 2012; 21: 614-25.

17. Wang G, Fersht AR. Multisite aggregation of p53 and implications for drug rescue. Proc Natl Acad Sci U S A. 2017; 114: E2634-43.

18. Alexandrova EM, Yallowitz AR, Li D, Xu S, Schulz R, Proia DA, Lozano G, Dobbelstein M, Moll UM. Improving survival by exploiting tumour dependence on stabilized mutant p53 for treatment. Nature. 2015; 523: 352-6.

19. Bauer MR, Joerger AC, Fersht AR. 2- Sulfonylpyrimidines: mild alkylating agents with anticancer activity toward p53compromised cells. Proc Natl Acad Sci U S A. 2016; 113: E5271-80.

20. Bykov VJ, Issaeva N, Shilov A, Hultcrantz M, Pugacheva E, Chumakov P, Bergman J, Wiman KG, Selivanova G. Restoration of the tumor suppressor function to mutant p53 by a low-molecular-weight compound. Nat Med. 2002; 8: 282-8.

21. Lambert JM, Moshfegh A, Hainaut P, Wiman KG, Bykov VJ. Mutant p53 reactivation by PRIMA-1MET induces multiple signaling pathways converging on apoptosis. Oncogene. 2010; 29: 1329-38.
22. Roh JL, Kang SK, Minn I, Califano JA, Sidransky D, Koch WM. p53- Reactivating small molecules induce apoptosis andenhance chemotherapeutic cytotoxicity in head and neck squamous cell carcinoma. Oral Oncol. 2011; 47: 8-15.

23. Liu X, Wilcken R, Joerger AC, Chuckowree IS, Amin J, Spencer J, Fersht AR. Small molecule induced reactivation of mutant p53 in cancer cells. Nucleic Acids Res. 2013; 41: 6034-44.

24. Bykov VJ, Zhang Q, Zhang M, Ceder S, Abrahmsen L, Wiman KG. Targeting of mutant p53 and the cellular redox balance by APR-246 as a strategy for efficient cancer therapy. Front Oncol. 2016; 6: 21.

25. Lambert JM, Gorzov P, Veprintsev DB, Söderqvist M, Segerbäck D, Bergman J, Fersht AR, Hainaut P, Wiman KG, Bykov VJ. PRIMA-1 reactivates mutant $\mathrm{p} 53$ by covalent binding to the core domain. Cancer Cell. 2009; 15: 376-88.

26. Hientz K, Mohr A, Bhakta-Guha D, Efferth T. The role of p53 in cancer drug resistance and targeted chemotherapy. Oncotarget. 2017; 8: 8921-46. https://doi.org/10.18632/ oncotarget. 13475.

27. Peng X, Zhang MQ, Conserva F, Hosny G, Selivanova G, Bykov VJ, Arnér ES, Wiman KG. APR-246/PRIMA-1MET inhibits thioredoxin reductase 1 and converts the enzyme to a dedicated NADPH oxidase. Cell Death Dis. 2013; 4: e881.

28. Shalom-Feuerstein R, Serror L, Aberdam E, Müller FJ, van Bokhoven $\mathrm{H}$, Wiman KG, Zhou H, Aberdam D, Petit I. Impaired epithelial differentiation of induced pluripotent stem cells from ectodermal dysplasia-related patients is rescued by the small compound APR-246/PRIMA-1MET. Proc Natl Acad Sci U S A. 2013; 110: 2152-6.

29. Deben C, Lardon F, Wouters A, Op de Beeck K, Van den Bossche J, Jacobs J, Van Der Steen N, Peeters M, Rolfo C, Deschoolmeester V, Pauwels P. APR-246 (PRIMA(MET)) strongly synergizes with AZD2281 (olaparib) induced PARP inhibition to induce apoptosis in non-small cell lung cancer cell lines. Cancer Lett. 2016; 375: 313-22.

30. Tessoulin B, Descamps G, Moreau P, Maïga S, Lodé L, Godon C, Marionneau-Lambot S, Oullier T, Le Gouill S, Amiot M, Pellat-Deceunynck C. PRIMA-1Met induces myeloma cell death independent of p53by impairing the GSH/ROS balance. Blood. 2014; 124: 1626-36.

31. Dulaney C, Marcrom S, Stanley J, Yang ES. Poly (ADPribose) polymerase activity and inhibition in cancer. Semin Cell Dev Biol. 2017; 63: 144-53.

32. Lord CJ, Ashworth A. PARP inhibitors: synthetic lethality in the clinic. Science. 2017; 355: 1152-8.

33. O'Shaughnessy J, Osborne C, Pippen JE, Yoffe M, Patt D, Rocha C, Koo IC, Sherman BM, Bradley C. Iniparib plus chemotherapy in metastatic triple-negative breast cancer. N Engl J Med. 2011; 364: 205-14.

34. Engert F, Schneider C, Wei $\beta$ LM, Probst M, Fulda S. PARP inhibitors sensitize Ewing sarcoma cells to 
temozolomide-induced apoptosis via the mitochondrial pathway. Mol Cancer Ther. 2015; 14: 2818-30.

35. Hsu HW, de Necochea-Campion R, Williams V, DuerksenHughes PJ, Simental AA Jr, Ferris RL, Chen CS, Mirshahidi S. Linifanib (ABT-869), enhances cytotoxicity with poly (ADP-ribose) polymerase inhibitor, veliparib (ABT-888), in head and neck carcinoma cells. Oral Oncol. 2014; 50: 662-9.

36. Wang Z, Jiang H, Chen S, Du F, Wang X. The mitochondrial phosphatase PGAM5 functions at the convergence point of multiple necrotic death pathways. Cell. 2012; 148: 228-43.

37. Degterev A, Hitomi J, Germscheid M, Chen IL, Korkina O, Teng X, Abbott D, Cuny GD, Yuan C, Wagner G, Hedrick SM, Gerber SA, Lugovskoy A, et al. Identification of RIP1 kinase as a specific cellular target of necrostatins. Nat Chem Biol. 2008; 4: 313-21.

38. Russo D, Ottaggio L, Foggetti G, Masini M, Masiello P, Fronza G, Menichini P. PRIMA-1 induces autophagy in cancer cells carrying mutant or wild type p53. Biochim Biophys Acta. 2013; 1833: 1904-13.

39. Kaminska KK, Bertrand HC, Tajima H, Stafford WC, Cheng Q, Chen W, Wells G, Arner ES, Chew EH. Indolin-2one compounds targeting thioredoxin reductase as potential anticancer drug leads. Oncotarget. 2016; 7: 40233-51. https://doi.org/10.18632/oncotarget.9579.

40. Xu HG, Zhai YX, Chen J, Lu Y, Wang JW, Quan CS, Zhao RX, Xiao X, He Q, Werle KD, Kim HG, Lopez R, Cui R, et al. LKB1 reduces ROS-mediated cell damage via activation of p38. Oncogene. 2015; 34: 3848-59.

41. Zeng Q, Li S, Chepeha DB, Giordano TJ, Li J, Zhang H, Polverini PJ, Nor J, Kitajewski J, Wang CY. Crosstalk between tumor and endothelial cells promotes tumor angiogenesis by MAPK activation of Notch signaling. Cancer Cell. 2005; 8: 13-23.

42. Leroy B, Ballinger ML, Baran-Marszak F, Bond GL, Braithwaite A, Concin N, Donehower LA, El-Deiry WS, Fenaux P, Gaidano G, Langerød A, Hellstrom-Lindberg E, Iggo R, et al. Recommended guidelines for validation, quality control, and reporting of TP53 variants in clinical practice. Cancer Res. 2017; 77: 1250-60.

43. Vogel SM, Bauer MR, Joerger AC, Wilcken R, Brandt T, Veprintsev DB, Rutherford TJ, Fersht AR, Boeckler FM. Lithocholic acid is an endogenous inhibitor of MDM4 and MDM2. Proc Natl Acad Sci U S A. 2012; 109: 16906-10.

44. Arihara Y, Takada K, Kamihara Y, Hayasaka N, Nakamura H, Murase K, Ikeda H, Iyama S, Sato T, Miyanishi K, Kobune M, Kato J. Small molecule CP-31398 induces reactive oxygen species-dependent apoptosis in human multiple myeloma. Oncotarget. 2017; 8: 65889-99. https:// doi.org/10.18632/oncotarget.19508.

45. Rieber M, Strasberg-Rieber M. Hypoxia, Mn-SOD and $\mathrm{H}(2) \mathrm{O}(2)$ regulate $\mathrm{p} 53$ reactivation and PRIMA-1 toxicity irrespective of p53 status in human breast cancer cells. Biochem Pharmacol. 2012; 84: 1563-70.

46. Fong PC, Boss DS, Yap TA, Tutt A, Wu P, MerguiRoelvink M, Mortimer P, Swaisland H, Lau A, O'Connor MJ, Ashworth A, Carmichael J, Kaye SB, et al. Inhibition of poly (ADP-ribose) polymerase in tumors from BRCA mutation carriers. N Engl J Med. 2009; 361: 123-34.

47. Cebula M, Schmidt EE, Arnér ES. TrxR1 as a potent regulator of the Nrf2-Keap1 response system. Antioxid Redox Signal. 2015; 23: 823-53.

48. van der Wijst MG, Huisman C, Mposhi A, Roelfes G, Rots MG. Targeting Nrf2 in healthy and malignant ovarian epithelialcells: protection versus promotion. Mol Oncol. 2015; 9: 1259-73.

49. Sakurai A, Nishimoto M, Himeno S, Imura N, Tsujimoto M, Kunimoto M, Hara S. Transcriptional regulation of thioredoxin reductase 1 expression by cadmium in vascular endothelial cells: role of NF-E2-related factor-2. J Cell Physiol. 2005; 203: 529-37.

50. Trachootham D, Alexandre J, Huang P. Targeting cancer cells by ROS-mediated mechanisms: a radical therapeutic approach? Nat Rev Drug Discov. 2009; 8: 579-91.

51. Wallace DC. Mitochondria and cancer. Nat Rev Cancer. 2012; 12: 685-98.

52. Costa A, Scholer-Dahirel A, Mechta-Grigoriou F. The role of reactive oxygen species and metabolism on cancer cells and their microenvironment. Semin Cancer Biol. 2014; 25: 23-32.

53. Fribley A, Zeng Q, Wang CY. Proteasome inhibitor PS341 induces apoptosis through induction of endoplasmic reticulum stress- reactive oxygen species in head and neck squamous cell carcinoma cells. Mol Cell Biol. 2004; 24: 9695-704.

54. Junk DJ, Vrba L, Watts GS, Oshiro MM, Martinez JD, Futscher BW. Different mutant/wild-type p53 combinations cause a spectrum of increased invasive potential in nonmalignant immortalized human mammary epithelial cells. Neoplasia. 2008; 10: 450-61.

55. Anestal K, Arner ES. Rapid induction of cell death by selenium-compromised thioredoxin reductase 1 but not by the fully active enzyme containing selenocysteine. J Biol Chem. 2003; 278: 15966-72. 